\title{
FDG avid cerebellar atypical meningioma masquerading as solitary brain metastases in a recently diagnosed breast malignancy: a toss between MR and CT
}

\author{
Shanmuga Sundaram Palaniswamy and Padma Subramanyam ${ }^{*}$ [D
}

\begin{abstract}
Background: SUV Max is a glycolytic index obtained from PET imaging, relates to tumour cell proliferation. FDG uptake (i.e. SUV max) is found to be high in aggressive tumours and is used to identify malignant from benign pathologies. Meningiomas are intracranial tumours which display varying grades of FDG avidity based on its biological aggressiveness. Benign grade I meningiomas are FDG non-avid, while the rest of the typical and atypical meningiomas show varying degrees of FDG avidity. Uptake of FDG can be high in certain infectious and inflammatory brain etiologies and pose a diagnostic challenge in differentiating benign brain lesions from neoplasms. MRI is the preferred modality for accurately identifying meningiomas, providing superior contrast differentiation and its ability to differentiate extra-axial from intra-axial brain lesions. CT is said to be superior in specific types of meningioma where there is calcification and adjacent changes in calvarium. Although typical meningiomas have characteristic MRI features, care must be taken to avoid misleading diagnosis between brain tumours and atypical meningiomas.
\end{abstract}

Case presentation: We are presenting a recently diagnosed case of invasive breast carcinoma (Ca) referred for staging by PET/MR imaging. Based on atypical DWI and ADC map findings, MRI falsely reported an atypical meningioma as a brain metastasis. Abnormal intense FDG uptake was noted in a well-defined homogeneously enhancing mass lesion in posterior fossa in left paramedian aspect and broad base to left transverse sinus protruding into left cerebellar hemisphere. Atypical meningioma Grade III, i.e. papillary meningioma was later histologically proven.

Conclusions: We wish to highlight the inconsistency of DWI and ADC map MR findings in papillary meningioma masquerading as solitary brain metastases in a Ca breast patient on 18F FDG PET/MR imaging. From an imaging standpoint, it is important to recognize the variable and pleomorphic features exhibited by meningiomas in MR based on atypical location, histological subtypes, and biologic behaviours. Further FDG PET was incremental in displaying a high SUV max indicating biologic aggressiveness of lesion and correlating with the CT diagnosis of papillary meningioma.

Keywords: Papillary meningioma, PET/MR, PET/CT, Brain metastases, ADC map, Case report

*Correspondence: drpadmasundaram@gmail.com

Department of Nuclear Medicine and Molecular Imaging, Amrita Institute of Medical Sciences, Amrita Vishwa Vidypeetham, Cochin, Kerala 6802041, India

\section{Background}

The tentative diagnosis of brain lesions like meningioma and many other benign, malignant tumours can be made by contrast enhanced MRI. MRI is also used for long term follow-up of brain tumours because of exquisite soft tissue differentiation. Combined use of FDG PET/MR 
provides additional functional information apart from exact tumour delineation, assessment of tumour growth, and pathophysiological parameters. Contrast-enhanced CT is performed as an alternate in those patients contraindicated for MRI. Meningiomas appear as broad-base dural hemispheric or oval lesions, attached to the dura mater. They most frequently occur supratentorially at the calvaria or the skull base meninges, along the falx and in the parafalcine location, but they can also be found attached to the tentorium, in the cerebellopontine angle, within the optic nerve sheath, or occurring intraventricularly [1]. On CT, meningiomas usually appear isodense but can occasionally be hyperdense or slightly hypodense compared with brain tissue. CT is more sensitive than MRI in detecting psammomatous calcifications in the tumour, which is seen in approximately $25 \%$ of meningiomas. On MRI, meningiomas typically present isointense to the cortex on T1- and T2-weighted sequences and with typically a strong homogeneous enhancement following administration of gadolinium contrast. They show varied DWI signal characteristics and ADC values. A variety of intracranial lesions may radiologically mimic meningioma. Studies show that tumour location, size, and growth pattern are taken into consideration for differential diagnosis of meningiomas [1]. If located at the skull base and especially at the cerebellopontine angle, meningiomas must be distinguished from vestibular schwannomas and neoplastic meningitis, none of them in fact clinically relevant in this patient. This case is being presented to highlight that MR findings can confound identification of certain atypical brain lesions. It is important to identify co-existing disease entities that call for different management especially in the background of invasive ductal carcinoma in a young patient. If one proceeds with the MR diagnosis alone, it could have led to mismanagement in this patient. The unusual location of a solitary large brain lesion along with atypical MR imaging characteristics is a challenge to the radiologist.

\section{Case presentation}

34-year-old lady, a known case of invasive right ductal carcinoma (pT1N0M0), post-mastectomy was referred to us for staging by whole-body FDG PET/MR. $1 \mathrm{mCi} / \mathrm{kg}$ body weight 18F FDG was injected intravenously in euglycemic status. An hour later, whole-body (Head-mid thigh) and dedicated brain images were acquired (with gadolinium contrast) on Biograph mMR, simultaneous PETMR scanner. Patient was clinically asymptomatic. There was no residual breast lesion or nodal disease on PET/MR imaging. However, abnormal FDG uptake was noted in a well-defined homogeneously enhancing mass lesion in posterior fossa in left paramedian aspect and broad based to left transverse sinus protruding into left cerebellar hemisphere (SUV Max $8.8 \mathrm{~g} / \mathrm{ml}$ ) (Fig. 1). Postcontrast T1W1 image of lesion showed homogenous enhancement, hyperintense on T2 weighted images, DWI revealed isointense to slight hypointensity lesion with predominantly low signal (thick arrow) and scattered hyperintensities (thin arrow) on ADC map unlike other atypical meningioma. Surrounding perilesional edema was noted. MRI findings were consistent with solitary brain metastases. CT raised another possibility of extra-axial cerebellar meningioma. Histopathology was conclusive and revealed characteristic perivascular pseudopapillary pattern suggesting papillary meningioma [2]. In a significant subset of patients especially those with higher WHO grades, where surgical approach is difficult or may be incomplete, adjuvant therapy regimens need to be applied. Our patient underwent gross total resection of brain tumour. Adjuvant chemotherapy was also initiated 6 weeks post-operatively, based on the histopathological diagnosis of primary invasive ductal carcinoma.

\section{Discussion}

Meningiomas arise from meningothelial cells of the arachnoid membranes. They are the most common primary intracranial neoplasms and represent about $20 \%$ of all intracranial tumours with female predominance. They are slow growing tumours, usually diagnosed in the third decade of life. According to World Health Organization, WHO criteria, meningiomas are classified into Grade 1 (benign), II (atypical), and III (anaplastic). FDG PET is useful in diagnosing cerebral tumour recurrence from radiation necrosis with a fair degree of confidence. However, normal glucose substrate in cerebral and cerebellar tissue is a major drawback. It falters in delineating slow growing tumours like meningiomas which may exhibit moderate FDG avidity due to poor target to non-target delineation. So, its ability to differentiate brain metastases from Grade II/III meningiomas or infective/inflammatory brain pathologies is challenging. Neuroimaging features, such as heterogeneous appearance, heterogeneous enhancement, marked perilesional edema, irregular cerebral surface, mushrooming on the outer edge of the lesion, and bone destruction, are not unique or reliable for diagnosing atypical/malignant meningiomas [3]. Reports also say that the accuracy and threshold ADC to distinguish between benign and atypical/malignant meningiomas has not been established [3]. The simultaneous occurrence of aggressive variant of meningioma and breast cancer with or without brain metastasis is rare [4]. Once histologically confirmed PET serves as useful yardstick in prognosticating and therapy response assessment of meningiomas. Studies have shown good correlation of FDG uptake to the proliferative potential of tumours. 


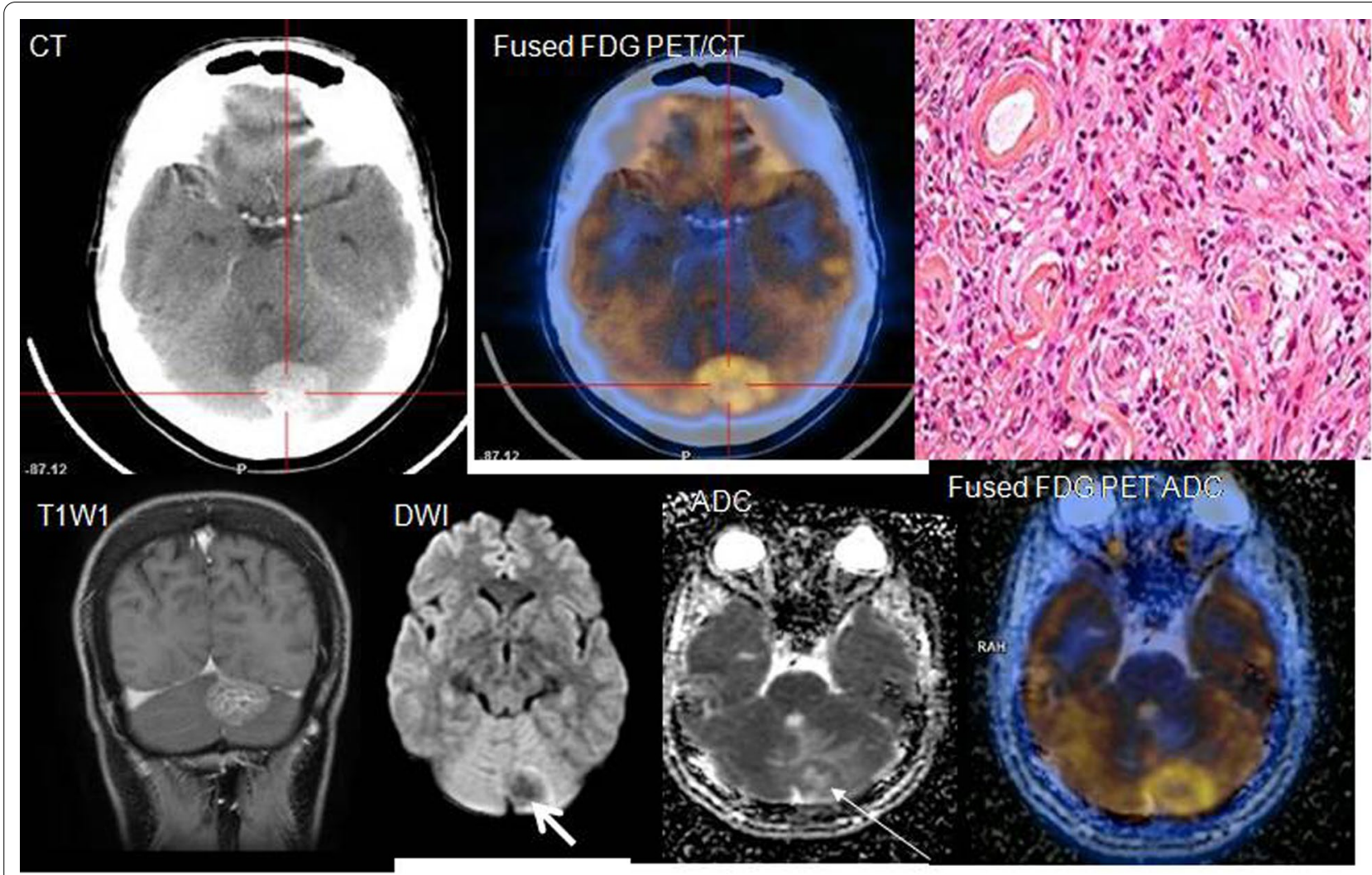

Fig. 1 Top row, right to left: transaxial brain CECT, fused FDG PET/CT transaxial brain image and histology of papillary meningioma. Bottom row: coronal MR (T1W1), DWI, ADC and fused (FDG PET/MR with ADC map) images. Post-contrast CT showed homogenous enhancement of extra-axial lesion (crosshair). Fused FDG PET/CT image showed increased FDG uptake consistent with the cerebellar lesion. Histopathology was conclusive and revealed characteristic perivascular pseudopapillary pattern suggesting papillary meningioma (WHO grade III). T1W1 image confirms homogenous enhancement of the lesion. DWI revealed isointense to slight hypointense lesion with predominantly low signal (thick arrow) and scattered hyperintensities (thin arrow) on ADC map unlike other atypical meningioma. Surrounding perilesional edema was noted. An MRI imaging diagnosis of solitary brain metastasis was made. But CT raised possibility of extra-axial cerebellar meningioma

Atypical meningiomas exhibit lower ADC than benign ones on MRI [5], like our case.

\section{Conclusions}

Uptake of ${ }^{18} \mathrm{~F}$ FDG correlates significantly with the WHO grade in meningiomas. Papillary meningioma, classified as WHO Grade III tumours, are extremely rare meningeal tumours that account for $1.0-2.5 \%$ of all meningiomas. Although typical meningiomas have characteristic MR findings, there are multiple atypical variants that masquerade as metastatic deposits. Papillary meningioma are aggressive and display high recurrence rates. From an imaging standpoint, it is important to recognize the variable and pleomorphic features exhibited by meningiomas based on atypical location, histological subtypes, and biologic behaviours. Histological confirmation is mandatory in such cases whenever possible before actual treatment is meted out.

\section{Abbreviations}

SUV max: Standardized uptake value maximum; MRI: Magnetic resonance imaging; 18F FDG PET: Fluorine Fluorodeoxyglucose Positron emission tomography; DWI: Diffusion-weighted imaging; ADC map: Attenuation diffusion coefficient map.

\section{Acknowledgements}

None.

\section{Authors' contributions}

SS, SP conceived the idea and contributed to the work up and draft of the manuscript. S.S. and S.P. contributed to the proofreading and finalization of the manuscript; and provided the patient related information; SS, SP interpreted the imaging findings as well as their interpretation. Both authors have read and approved the final manuscript.

\section{Funding}

No funding sources or grants.

\section{Availability of data and materials}

Not applicable. 


\section{Declarations}

\section{Ethics approval and consent to participate}

PET is an established routine imaging procedure, ethics approval not needed. Patient has given written consent for undergoing both sets of imaging.

\section{Consent for publication}

Patient has given written consent for this publication. This was obtained directly from patient being an adult (legal age).

\section{Competing interests}

None.

Received: 13 August 2021 Accepted: 13 November 2021

Published online: 26 November 2021

\section{References}

1. Zimny A, Sasiadek M (2011) Contribution of perfusion-weighted magnetic resonance imaging in the differentiation of meningiomas and other extra-axial tumors: case reports and literature review. J Neurooncol 103(3):777-83

2. Avninder S, Vermani S, Shruti S, Chand K (2010) Papillary meningioma: a rare but distinct variant of malignant meningioma. Diagn Pathol 19(2):3

3. Modha A, Gutin PH (2005) Diagnosis and treatment of atypical and anapastic meningiomas: a review. Neurosurgery 57:538-550

4. Seckin H, Yigitkanli K, llhan O, Han U, Bavbek M (2006) Breast carcinoma metastasis and meningioma. A case report. Surg Neurol 66(3):324-327

5. Nagar VA, Ye JR, Ng WH, Chan YH, Hui F, Lee CK (2008) Diffusion-weighted MR imaging: diagnosing atypical or malignant meningiomas and detecting tumor dedifferentiation. Am J Neuroradiol 29(6):1147-1152

\section{Publisher's Note}

Springer Nature remains neutral with regard to jurisdictional claims in published maps and institutional affiliations.

\section{Submit your manuscript to a SpringerOpen ${ }^{\circ}$ journal and benefit from:}

- Convenient online submission

- Rigorous peer review

- Open access: articles freely available online

- High visibility within the field

- Retaining the copyright to your article 